\title{
Enzyme Architecture: Erection of Active Orotidine 5'- Monophosphate Decarboxylase by Substrate-Induced Conformational Changes
}

Archie C. Reyes, Tina L. Amyes, and John P. Richard*(i)

Department of Chemistry, University at Buffalo, SUNY, Buffalo, New York 14260-3000, United States

Supporting Information

ABSTRACT: Orotidine 5'-monophosphate decarboxylase (OMPDC) catalyzes the decarboxylation of 5fluoroorotate (FO) with $k_{\text {cat }} / K_{\mathrm{m}}=1.4 \times 10^{-7} \mathrm{M}^{-1} \mathrm{~s}^{-1}$. Combining this and related kinetic parameters shows that the $31 \mathrm{kcal} / \mathrm{mol}$ stabilization of the transition state for decarboxylation of OMP provided by OMPDC represents the sum of 11.8 and $10.6 \mathrm{kcal} / \mathrm{mol}$ stabilization by the substrate phosphodianion and the ribosyl ring, respectively, and an $8.6 \mathrm{kcal} / \mathrm{mol}$ stabilization from the orotate ring. The transition state for OMPDC-catalyzed decarboxylation of FO is stabilized by $5.2,7.2$, and $9.0 \mathrm{kcal} / \mathrm{mol}$, respectively, by $1.0 \mathrm{M}$ phosphite dianion, D-glycerol 3phosphate and D-erythritol 4-phosphate. The stabilization is due to the utilization of binding interactions of the substrate fragments to drive an enzyme conformational change, which locks the orotate ring of the whole substrate, or the substrate pieces in a caged complex. We propose that enzyme-activation is a possible, and perhaps probable, consequence of any substrate-induced enzyme conformational change.

$\mathrm{T}$ he underlying cause for enzymatic catalysis is stabilization of the transition state by interactions with the protein catalyst. ${ }^{1}$ Orotidine $5^{\prime}$-monophosphate decarboxylase (OMPDC) affects a $31 \mathrm{kcal} / \mathrm{mol}$ stabilization of the transition state for decarboxylation of orotidine $5^{\prime}$-monophosphate (OMP) to give uridine $5^{\prime}$-monophosphate (UMP), ${ }^{2}$ by a stepwise mechanism through a UMP carbanion intermediate (Scheme 1). ${ }^{3}$ This transition state stabilization has been modeled in computational studies. ${ }^{4}$ What has not been fully modeled is the extraordinary specificity of OMPDC in binding

Scheme 1. Substrate Fragments of OMP that Provide the Binding Energy Utilized in the Stabilization of the UMP Carbanion Intermediate of OMPDC-Catalyzed Decarboxylation.

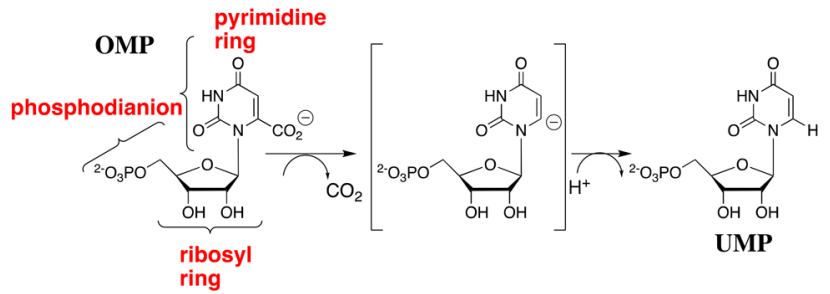

the decarboxylation transition state with a higher affinity (31 $\mathrm{kcal} / \mathrm{mol}$ ) compared with the substrate OMP $(8 \mathrm{kcal} / \mathrm{mol}))^{2,5}$

Binding interactions between OMPDC and the phosphodianion of OMP provide 12 of the $31 \mathrm{kcal} / \mathrm{mol}$ transition state stabilization. ${ }^{6}$ These interactions do not only anchor OMP to the protein, because eliminating the anchoring connection results in only a $4 \mathrm{kcal} / \mathrm{mol} \mathrm{decrease,} \mathrm{to} 8 \mathrm{kcal} / \mathrm{mol}$, in the stabilization of the transition state for decarboxylation of the truncated substrate piece 1- $(\beta$-D-erythrofuranosyl)orotate (EO) from interactions with 1.0 M phosphite dianion (Figure 1A). ${ }^{6,7}$
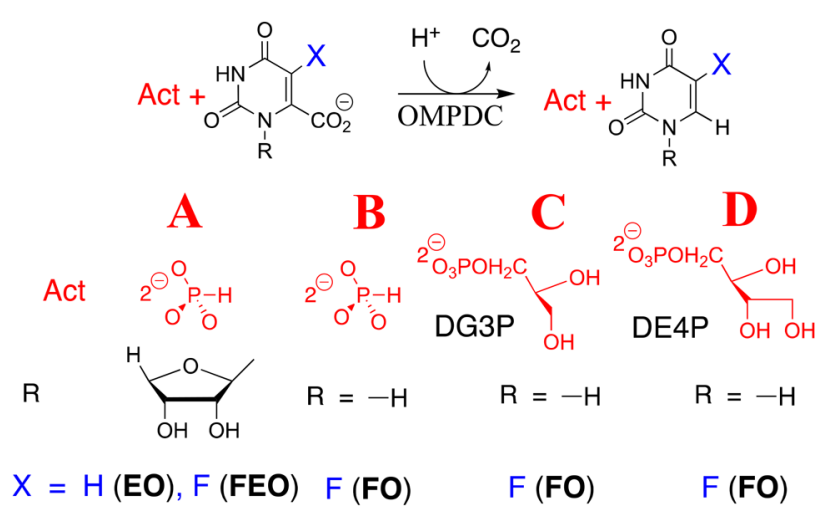

Figure 1. Activation of OMPDC-catalyzed decarboxylation reactions. (A) Activation of decarboxylation of $\mathbf{E O}$ and $\mathbf{F E O}$ by $\mathrm{HPO}_{3}{ }^{2-6,10}$ (B) Activation of decarboxylation of $\mathbf{F O}$ by $\mathrm{HPO}_{3}{ }^{2-}$. (C, D) Activation of decarboxylation of FO by D-glycerol 3-phosphate (DG3P) and Derythritol 4-phosphate (DE4P), respectively.

This activation is due to the utilization of binding energy from interactions between OMPDC and activator (Act, Figure 1) to drive a complex conformational change from inactive open $\operatorname{OMPDC}\left(\mathbf{E}_{\mathbf{O}}\right)$ to the active closed caged complex $\left(\mathbf{E}_{\mathbf{C}}\right),{ }^{8}$ where $\mathrm{E}_{\mathrm{C}}$ is stabilized relative to $\mathrm{E}_{\mathrm{O}}$ by interactions between dianions and the side chains of Q215, Y217, and R235.

Figure 2A shows a representation of the open form of OMPDC determined for unliganded OMPDC from yeast $\left(\mathbf{E}_{\mathbf{O}}\right.$, Scheme 2), with a hypothetical 6-aza uridine 5'-monophosphate (azaUMP) ligand inserted at the position determined for the OMPDC-azaUMP complex (Figure 2B). ${ }^{11}$ Many ligands induce a large conformational change in OMPDC that is driven by the development of strong protein-

Received: August 20, 2017

Published: October 23, 2017 


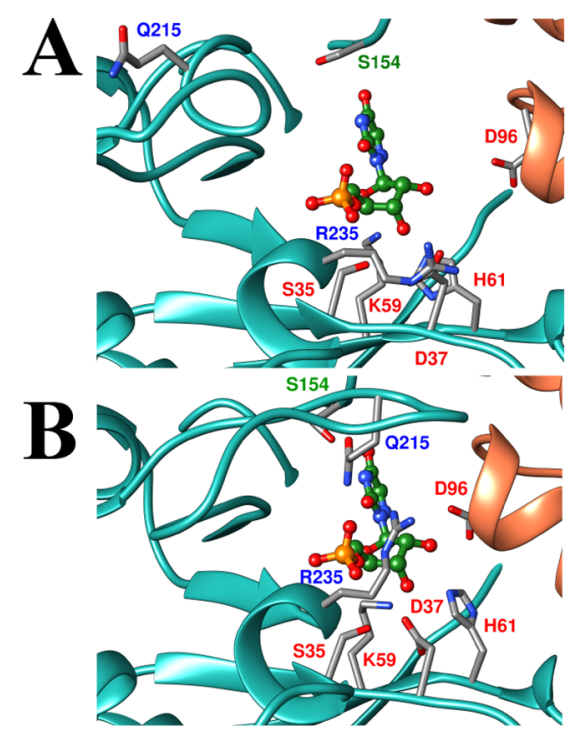

Figure 2. Representations of the open $\mathbf{E}_{\mathbf{O}}$ (PDB entry $3 \mathrm{GDK}$ ) and the closed, liganded, (B, 3GDL) forms of yeast OMPDC. ${ }^{11}$ The azaUMP ligand is placed at structure $\mathrm{A}$ at the position determined for structure $\mathrm{B}$. The ligand is stabilized by interactions from the side chains of R235 and Q215 with the phosphodianion, and of the D96, H61, D37 and K59 with the ribosyl hydroxyls.

Scheme 2. The Role of an Activator-Driven Conformational Change in Catalysis by OMPDC.

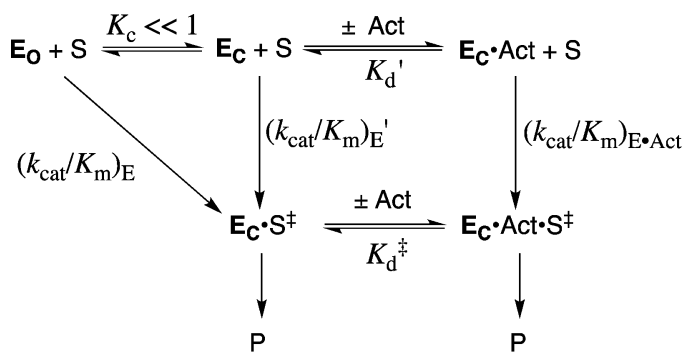

ligand interactions. ${ }^{12}$ The interactions between the protein and the phosphodianion, or the ribosyl hydroxyls, which develop at the complex to the tight-binding inhibitor azaUMP [or to substrate analogs], ${ }^{12}$ are illustrated in Figure $2 \mathrm{~B}$ for the closed form of OMPDC $\left(\mathbf{E}_{\mathbf{C}}\right) .{ }^{11}$ Our model (Scheme 2) requires that the binding energy from all interactions that drive the conformational change from $\mathbf{E}_{\mathrm{O}}$ to $\mathrm{E}_{\mathrm{C}}$ activate OMPDC for catalysis, as has been demonstrated for protein-dianion interactions, ${ }^{6,10,13}$ because these interactions are only fully expressed at the decarboxylation transition state. This prediction is confirmed here by the observation that binding interactions between OMPDC and either phosphite dianion (Figure 1B) or sugar hydroxyls (Figure 1C and 1D) activate OMPDC for catalysis of decarboxylation of the definitive truncated substrate, 5-fluoroorotate (FO).

The slow decarboxylation of FO to form 5-fluorouracil (FU) catalyzed by yeast OMPDC was monitored by HPLC analyses, as described in the Supporting Information (SI). Observed firstorder rate constants $k_{\mathrm{obs}}=v /[\mathrm{E}]$ for the decarboxylation of FO (5 or $10 \mathrm{mM}$ ) catalyzed by $0.7 \mathrm{mM}$ OMPDC at $25^{\circ} \mathrm{C}$ were determined from the initial reaction velocity $v$ during the first $0.01 \%$ reaction, over a two-week reaction time, during which OMPDC maintained full activity. The second-order rate constant $\left(k_{\text {cat }} / K_{\mathrm{m}}\right)_{\mathrm{o}}=(1.4 \pm 0.1) \times 10^{-7} \mathrm{M}^{-1} \mathrm{~s}^{-1}$ for
OMPDC-catalyzed decarboxylation of FO (Table 1) was determined as the slope of the linear plot of $k_{\mathrm{obs}}=v[\mathrm{E}]$

Table 1. Contribution of the IBE from Substrate Fragments to the $31 \mathrm{kcal} / \mathrm{mol} \mathrm{IBE}$ of $\mathrm{OMP}^{2}$ for $\mathrm{OMPDC}^{a}$

\begin{tabular}{llll} 
Substrate & $k_{\text {cat }} / K_{\mathrm{m}}\left(\mathrm{M}^{-1} \mathrm{~s}^{-1}\right)^{b}$ & \multicolumn{1}{c}{ Fragment $^{c}$} & $\begin{array}{c}\text { Fragment IBE } \\
(\mathrm{kcal} / \mathrm{mol})^{d}\end{array}$ \\
FO & $(1.4 \pm 0.1) \times 10^{-7 e}$ & & \\
Orotate & $3 \times 10^{-10 f}$ & $\mathrm{H}$ & 0 \\
EO & 0.02 & D-Erythrose & 10.6 \\
OMP & $1 \times 10^{7}$ & $\begin{array}{c}\text { Ribose 5'- } \\
\text { Phosphate }\end{array}$ & $10.6+11.8$
\end{tabular}

${ }^{a}$ At $25{ }^{\circ} \mathrm{C}$, pH 7.0 and $I=0.14-0.15(\mathrm{NaCl}) .{ }^{b}$ Second-order rate constant for decarboxylation catalyzed by OMPDC. ${ }^{c}$ Fragment attached to orotate. ${ }^{d}$ Contribution of fragment to the stabilization of the transition state for OMPDC catalyzed decarboxylation of OMP. ${ }^{e}$ Figure $3 \mathrm{~A} .{ }^{f}$ See text.
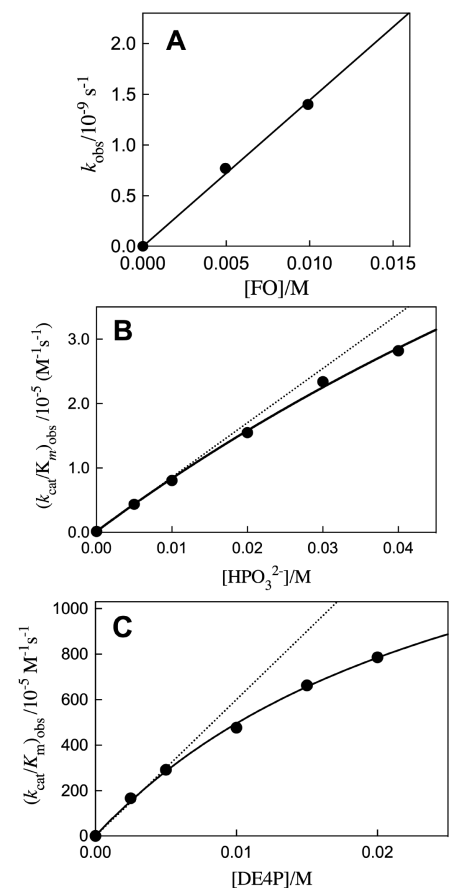

Figure 3. Plots of kinetic data for OMPDC-catalyzed decarboxylation of FO. (A) The dependence of $k_{\mathrm{obs}}=v[\mathrm{E}]$ on [FO]. (B) The dependence of $\left(k_{\text {cat }} / K_{\mathrm{m}}\right)_{\text {obs }}$ for OMPDC-catalyzed decarboxylation of FO on $\left[\mathrm{HPO}_{3}{ }^{2-}\right]$. (C) The dependence of $\left(k_{\mathrm{cat}} / K_{\mathrm{m}}\right)_{\mathrm{obs}}$ for OMPDCcatalyzed decarboxylation of FO on the concentration of DE4P.

against [FO] (Figure 3A). These apparent first-order rate constants from HPLC analyses are reproducible to better than $\pm 10 \%$. The 5 -F substituent provides strong stabilization of the UMP carbanion intermediate of OMPDC-catalyzed reactions. ${ }^{3 a, 10,13 b, 14}$ The value of $\left(k_{\text {cat }} / K_{\mathrm{m}}\right)_{\mathrm{o}}=3 \times 10^{-10} \mathrm{M}^{-1} \mathrm{~s}^{-1}$ for OMPDC-catalyzed decarboxylation of orotate (Table 1) was determined from $\left(k_{\mathrm{cat}} / K_{\mathrm{m}}\right)_{\mathrm{o}}=1.4 \times 10^{-7} \mathrm{M}^{-1} \mathrm{~s}^{-1}$ for decarboxylation of FO and a 500-fold effect of the 5-F substituent. This is a rough average of the 5-F effect on mutant OMPDC-catalyzed decarboxylation of OMP, when chemistry is strongly rate determining for both OMPDC-catalyzed reactions. 
A comparison of the second-order rate constants $k_{\mathrm{cat}} / K_{\mathrm{m}}$ for OMPDC-catalyzed decarboxylation of orotate, EO, and OMP (Table 1) shows that the ribosyl and phosphodianion fragments contribute 10.6 and $11.8 \mathrm{kcal} / \mathrm{mol}$ intrinsic binding energies, respectively, to stabilization of the transition state for OMPDCcatalyzed decarboxylation of OMP: the sum is $22.4 \mathrm{kcal} / \mathrm{mol}$ of the total $31 \mathrm{kcal} / \mathrm{mol}$ total intrinsic binding energy of OMP, leaving $8.6 \mathrm{kcal} / \mathrm{mol}$ for stabilization of the transition state by interactions with orotate. ${ }^{2}$ This provides a graphic and readily generalizable illustration of how enormous enzymatic rate accelerations may be obtained through recruitment of several modest, additive, binding energies of substrate fragments.

Figure 3B,C shows the effect of increasing $\left[\mathrm{HPO}_{3}{ }^{2-}\right]$ and [DE4P], respectively, on $\left(k_{\mathrm{cat}} / K_{\mathrm{m}}\right)_{\mathrm{obs}}$ for OMPDC-catalyzed decarboxylation of FO $(5 \mathrm{mM})$. These data were fit to eq 1 (Scheme $3 \mathrm{~A})$ to give the values for $\left(k_{\text {cat }} / K_{\mathrm{m}}\right)_{\mathrm{Act}}$ and $K_{\mathrm{d}}$ reported

Scheme 3. (a) Kinetic Scheme Used for the Derivation of eq 1. (b) Kinetic Scheme Used for the Derivation of eq 3.
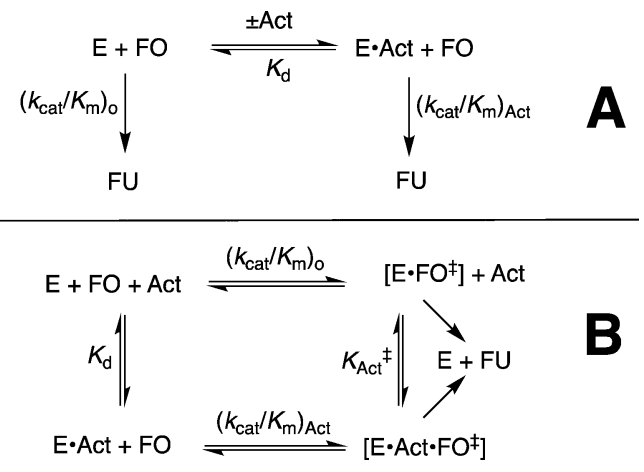

in Table 2. Table 2 reports values for $\left(k_{\mathrm{cat}} / K_{\mathrm{m}}\right)_{\text {Act }} / K_{\mathrm{d}}$ obtained as the slope of the linear correlations shown as dashed lines in Figure 3B,C. Data for the effect of increasing [D,LG3P] (Figure $\mathrm{S} 1 \mathrm{~A})$ and [LG3P] (Figure S1B) on $\left(k_{\mathrm{cat}} / K_{\mathrm{m}}\right)_{\mathrm{obs}}$ for OMPDCcatalyzed decarboxylation of FO were evaluated to give the kinetic parameters in Table 2 . The value of $\left(k_{\text {cat }} / K_{\mathrm{m}}\right)_{\text {Act }} / K_{\mathrm{d}}$ for OMPDC-catalyzed decarboxylation of DG3P was calculated from these kinetic parameters using eq 2 , where $\left(k_{\mathrm{Act}}\right)_{\mathrm{XG3P}}=$ $\left.\left[\left(k_{\mathrm{cat}} / K_{\mathrm{m}}\right)_{\mathrm{Act}} / K_{\mathrm{d}}\right)\right]_{\mathrm{XG} 3 \mathrm{P}}(\mathrm{X}=\mathrm{D}, \mathrm{L}$, or $\mathrm{D}, \mathrm{L})$. The intrinsic fragment binding energies (IBEs), determined using eq 3 (Scheme 3B), are reported in Table 2.

Phosphite dianion $(1.0 \mathrm{M})$ provides similar $5.0^{10}$ and 5.2 (Table 2) $\mathrm{kcal} / \mathrm{mol}$ stabilization, respectively, of the transition states for OMPDC-catalyzed decarboxylation of truncated substrates 1-( $\beta$-D-erythrofuranosyl)-5-fluoroorotate (FEO) and FO. The small apparent effect of the ribosyl group of
FEO on activation for decarboxylation shows that the effect of the dianion driven protein conformational change on the reactivity of the fluoroorotate ring is transmitted across both the ribosyl group and the vacant protein core. DG3P and DE4P provide 7.2 and $9.0 \mathrm{kcal} / \mathrm{mol}$ transition state stabilization, respectively, which corresponds to $c a .2 \mathrm{kcal} / \mathrm{mol}$ transition state stabilization/sugar hydroxyl. OMPDC shows specificity for activation by DG3P, because the IBE determined for LG3P is similar to that for $\mathrm{HPO}_{3}{ }^{2-}$ alone. The small activation of OMPDC-catalyzed decarboxylation of FO for a reaction in the presence of $40 \mathrm{mM}$ D-ribose 5'-phosphate (DR5P, SI) is consistent with a ligand IBE of only $3.1 \mathrm{kcal} / \mathrm{mol}$ (Table 2). This shows that a tight and precise fit of the ligand is required for strong transition state binding. ${ }^{10}$

$$
\begin{aligned}
&\left(k_{\mathrm{cat}} / K_{\mathrm{m}}\right)_{\mathrm{obs}}=\left(\frac{K_{\mathrm{d}}}{K_{\mathrm{d}}+[\text { Act }]}\right)\left(k_{\mathrm{cat}} / K_{\mathrm{m}}\right)_{\mathrm{o}}+\left(\frac{[\text { Act }]}{K_{\mathrm{d}}+[\text { Act }]}\right) \\
& \times\left(k_{\mathrm{cat}} / K_{\mathrm{m}}\right)_{\text {Act }} \\
&\left(k_{\text {Act }}\right)_{\mathrm{DG} 3 \mathrm{P}}=2\left(k_{\mathrm{Act}}\right)_{\mathrm{D}, \mathrm{LG} 3 \mathrm{P}}-\left(k_{\mathrm{Act}}\right)_{\mathrm{LG} 3 \mathrm{P}} \\
& K_{\text {Act }}^{\dagger}=\left(\frac{K_{\mathrm{d}}\left(k_{\mathrm{cat}} / K_{\mathrm{m}}\right)_{\mathrm{o}}}{\left(k_{\mathrm{cat}} / K_{\mathrm{m}}\right)_{\mathrm{Act}}}\right)
\end{aligned}
$$

These results provide strong support for the conclusion that each of the many OMPDC-substrate interactions, which stabilize the closed enzyme $\mathbf{E}_{\mathbf{C}}$ relative to the open enzyme $\mathbf{E}_{\mathbf{O}}$, contribute to activation of OMPDC for catalysis of decarboxylation of orotate and fluoroorotate rings. We conclude that these protein-ligand interactions act in concert to construct a tight, catalytically active form of OMPDC from the floppy open enzyme. These results provide a dramatic example of the effect of such preorganization of protein structure on enzyme activity. ${ }^{15}$

X-ray crystallographic analyses and other protocols for the evaluation of the role of conformational changes in enzyme catalysis, failed to suggest the activating nature of phosphodianion driven conformational changes in catalysis by triosephosphate isomerase, and other enzymes. ${ }^{7 b, 16}$ Our report of the, likewise, unrecognized activating role of protein sugarhydroxyl interactions in catalysis by OMPDC provides compelling motivation for a re-evaluation of the role of substrate-driven conformational changes in enzyme cataly$\operatorname{sis}^{1 \mathrm{l}, 8 \mathrm{a}, \mathrm{b}, 17}$ and, for experiments to test the hypothesis that many, or most, such protein conformational changes activate enzymes for catalysis of the reaction of their bound substrates.

\begin{tabular}{|c|c|c|c|c|}
\hline Activator & $\left(k_{\mathrm{cat}} / K_{\mathrm{m}}\right)_{\mathrm{Act}} \mathrm{M}^{-1} \mathrm{~s}^{-1 b}$ & $K_{\mathrm{d}}(\mathrm{M})^{c}$ & {$\left[\left(k_{\text {cat }} / K_{\mathrm{m}}\right)_{\mathrm{Act}}\right] / K_{\mathrm{d}} \mathrm{M}^{-2} \mathrm{~s}^{-1 d}$} & $\mathrm{IBE}(\mathrm{kcal} / \mathrm{mol})$ \\
\hline $\mathrm{HP}_{\mathrm{i}}^{f}$ & $(1.6 \pm 0.4) \times 10^{-4}$ & $0.18 \pm 0.05$ & $(8.4 \pm 0.4) \times 10^{-4}$ & 5.2 \\
\hline $\mathrm{D}, \mathrm{LG} 3 \mathrm{P}^{g}$ & $(7.0 \pm 0.7) \times 10^{-4}$ & $0.05 \pm 0.01$ & $(1.3 \pm 0.1) \times 10^{-2}$ & 6.8 \\
\hline LG3P $P^{g, h}$ & & & $(9.9 \pm 0.2) \times 10^{-4}$ & 5.2 \\
\hline $\mathrm{DG} \mathrm{P}^{i}$ & & & $(2.5 \pm 0.01) \times 10^{-2}$ & 7.2 \\
\hline $\mathrm{DE} 4 \mathrm{P}^{j}$ & $(1.9 \pm 0.2) \times 10^{-2}$ & $0.030 \pm 0.003$ & $(6.0 \pm 0.2) \times 10^{-1}$ & 9.0 \\
\hline DR5P $^{g}$ & & & $\approx 3 \times 10^{-5}$ & 3.1 \\
\hline
\end{tabular}

Experiments to probe the activation of OMPDC by substrate induced conformational changes have outpaced other mecha-

Table 2. Kinetic Parameters for Unactivated and Activated OMPDC-Catalyzed Decarboxylation of FO ${ }^{a}$

${ }^{a}$ At $25{ }^{\circ} \mathrm{C}$, pH 7.0 and $I=0.15(\mathrm{NaCl})$. The quoted errors are the standard deviations from the least-squares fits of the data. ${ }^{b}$ Second-order rate constant for breakdown of E·Act.FO to form FU. ${ }^{c}$ Dissociation constant for the activator. ${ }^{d}$ Determined as described in the text. ${ }^{e}$ Fragment intrinsic binding energy, calculated as $-R T \ln K^{\ddagger}$ (eq 3). ${ }^{f}$ Figure $3 \mathrm{~B} .{ }^{g}$ See SI. ${ }^{h}$ No detectable saturation of OMPDC. ${ }^{i}$ Calculated from eq 2 , where $\left(k_{\text {Act }}\right)=$ $\left[\left(k_{\text {cat }} / K_{\mathrm{m}}\right)_{\text {Act }}\right] / K_{\mathrm{d}}$ from Scheme $3 \mathrm{~A} .{ }^{j}$ Figure $3 \mathrm{C}$. 
nistic studies on this enzyme. Consequently, there is now strong evidence that the binding interactions of the nonreacting substrate parts are used to construct a protein cage that shows a high reactivity toward substrate decarboxylation. The results of our earlier work have emphasized the role the protein plays in strongly stabilizing the UMP carbanion intermediate relative to the carbon acid substrate. ${ }^{3}$ This suggests that an unusually strong stabilization of this carbanion by interaction with the cationic side chain of K93. ${ }^{4 c, 16 a}$ This single interaction seems unlikely to enable the entire rate acceleration, so that there remains much to be learned about the origin of the highreactivity of the caged OMPDC-substrate complex

\section{ASSOCIATED CONTENT}

\section{S Supporting Information}

The Supporting Information is available free of charge on the ACS Publications website at DOI: 10.1021/jacs.7b08897.

References for experimental procedures used to purify yeast OMPDC, and a description of the procedures for the assay of OMPDC-catalyzed decarboxylation of FO; kinetic data for the activation of OMPDC by D-ribose $5^{\prime}$ phosphate; effect on increasing [LG3P] and [D,LG3P], respectively on $\left(k_{\mathrm{cat}} / K_{\mathrm{m}}\right)_{\mathrm{obs}}$ for OMPDC-catalyzed decarboxylation of FO (PDF)

\section{AUTHOR INFORMATION}

\section{Corresponding Author}

*jrichard@buffalo.edu

\section{ORCID}

John P. Richard: 0000-0002-0440-2387

\section{Notes}

The authors declare no competing financial interest.

\section{ACKNOWLEDGMENTS}

This work was supported by grants GM116921 and GM39754 from the National Institutes of Health.

\section{REFERENCES}

(1) (a) Pauling, L. Nature 1948, 161, 707-709. (b) Amyes, T. L.; Richard, J. P. Biochemistry 2013, 52, 2021-2035.

(2) Radzicka, A.; Wolfenden, R. Science 1995, 267, 90-93.

(3) (a) Tsang, W.-Y.; Wood, B. M.; Wong, F. M.; Wu, W.; Gerlt, J. A.; Amyes, T. L.; Richard, J. P. J. Am. Chem. Soc. 2012, 134, 1458014594. (b) Amyes, T. L.; Wood, B. M.; Chan, K.; Gerlt, J. A.; Richard, J. P. J. Am. Chem. Soc. 2008, 130, 1574-1575.

(4) (a) Vardi-Kilshtain, A.; Doron, D.; Major, D. T. Biochemistry 2013, 52, 4382-4390. (b) Wu, N.; Mo, Y.; Gao, J.; Pai, E. F. Proc. Natl. Acad. Sci. U. S. A. 2000, 97, 2017-2022. (c) Warshel, A.; Strajbl, M.; Villa, J.; Florian, J. Biochemistry 2000, 39, 14728-14738. (d) Gao, J. Curr. Opin. Struct. Biol. 2003, 13, 184-192.

(5) Porter, D. J. T.; Short, S. A. Biochemistry 2000, 39, 11788-11800.

(6) Amyes, T. L.; Richard, J. P.; Tait, J. J. J. Am. Chem. Soc. 2005, 127, 15708-15709.

(7) (a) Spong, K.; Amyes, T. L.; Richard, J. P. J. Am. Chem. Soc. 2013, 135, 18343-18346. (b) Reyes, A. C.; Zhai, X.; Morgan, K. T.; Reinhardt, C. J.; Amyes, T. L.; Richard, J. P. J. Am. Chem. Soc. 2015, 137, 1372-1382.

(8) (a) Amyes, T. L.; Malabanan, M. M.; Zhai, X.; Reyes, A. C.; Richard, J. P. Protein Eng., Des. Sel. 2017, 30, 159-168. (b) Richard, J. P.; Amyes, T. L.; Goryanova, B.; Zhai, X. Curr. Opin. Chem. Biol. 2014, 21, 1-10. (c) Go, M. K.; Amyes, T. L.; Richard, J. P. Biochemistry 2009, 48, 5769-5778.

(9) (a) Amyes, T. L.; Ming, S. A.; Goldman, L. M.; Wood, B. M.; Desai, B. J.; Gerlt, J. A.; Richard, J. P. Biochemistry 2012, 51, 4630-
4632. (b) Goldman, L. M.; Amyes, T. L.; Goryanova, B.; Gerlt, J. A.; Richard, J. P. J. Am. Chem. Soc. 2014, 136, 10156-10165.

(10) Goryanova, B.; Spong, K.; Amyes, T. L.; Richard, J. P. Biochemistry 2013, 52, 537-546.

(11) Chan, K. K.; Wood, B. M.; Fedorov, A. A.; Fedorov, E. V.; Imker, H. J.; Amyes, T. L.; Richard, J. P.; Almo, S. C.; Gerlt, J. A. Biochemistry 2009, 48, 5518-5531.

(12) (a) Fujihashi, M.; Wei, L.; Kotra, L. P.; Pai, E. F. J. Mol. Biol. 2009, 387, 1199-1210. (b) Wittmann, J. G.; Heinrich, D.; Gasow, K.; Frey, A.; Diederichsen, U.; Rudolph, M. G. Structure 2008, 16, 82-92.

(13) (a) Goryanova, B.; Goldman, L. M.; Amyes, T. L.; Gerlt, J. A.; Richard, J. P. Biochemistry 2013, 52, 7500-7511. (b) Goryanova, B.; Amyes, T. L.; Gerlt, J. A.; Richard, J. P. J. Am. Chem. Soc. 2011, 133, $6545-6548$

(14) Goryanova, B.; Goldman, L. M.; Ming, S.; Amyes, T. L.; Gerlt, J. A.; Richard, J. P. Biochemistry 2015, 54, 4555-4564.

(15) Warshel, A. J. Biol. Chem. 1998, 273, 27035-27038.

(16) (a) Miller, B. G.; Hassell, A. M.; Wolfenden, R.; Milburn, M. V.; Short, S. A. Proc. Natl. Acad. Sci. U. S. A. 2000, 97, 2011-2016. (b) Ou, X.; Ji, C.; Han, X.; Zhao, X.; Li, X.; Mao, Y.; Wong, L.-L.; Bartlam, M.; Rao, Z. J. Mol. Biol. 2006, 357, 858-869. (c) Davenport, R. C.; Bash, P. A.; Seaton, B. A.; Karplus, M.; Petsko, G. A.; Ringe, D. Biochemistry 1991, 30, 5821-6. (d) Kholodar, S. A.; Allen, C. L.; Gulick, A. M.; Murkin, A. S. J. Am. Chem. Soc. 2015, 137, 2748-2756.

(17) Malabanan, M. M.; Amyes, T. L.; Richard, J. P. Curr. Opin. Struct. Biol. 2010, 20, 702-710. 